Bull. Austral. Math. Soc.

Vol. 54 (1996) [67-77]

\title{
PLURIHARMONIC SYMBOLS OF COMMUTING TOEPLITZ TYPE OPERATORS
}

YOUNG JOO LEE

\begin{abstract}
Certain Toeplitz type operators acting on the Bergman space $A^{1}$ of the unit ball are considered and pluriharmonic symbols of commuting Toeplitz type operators are characterised by using $\mathcal{M}$-harmonic function theory.
\end{abstract}

\section{INTRODUCtion AND Result}

Let $B$ be the unit ball of the $n$-dimensional complex space $\mathbb{C}^{n}$. The Bergman space $A^{p}(1 \leqslant p \leqslant \infty)$ is the closed subspace of the Lebesgue space $L^{p}=L^{p}(B, V)$ consisting of holomorphic functions where $V$ denotes the Lebesgue volume measure on $B$ normalised to have total mass 1 . Let $Q$ be the integral operator on $L^{1}$ defined by

$$
Q(\psi)(z)=\lambda_{n} \int_{B} \frac{\left(1-|w|^{2}\right)^{n+1}}{(1-\langle z, w\rangle)^{2 n+2}} \psi(w) d V(w) \quad(z \in B)
$$

for functions $\psi \in L^{1}$ where $\langle$,$\rangle is the ordinary Hermitian inner product on \mathbb{C}^{n}$ and $1 / \lambda_{n}=\int_{B}\left(1-|w|^{2}\right)^{n+1} d V(w)$. It is known that $Q$ is a bounded linear operator taking $L^{1}$ onto $A^{1}$. Moreover, $Q$ has the following reproducing properties:

$$
Q f=f \quad \text { and } \quad Q \bar{f}=\bar{f}(0)
$$

for functions $f \in A^{1}$. See [6, Chapter 7] for more informations on the operator $Q$ and related facts. For $u \in L^{\infty}$, the Toeplitz type operator $T_{u}$ with symbol $u$ is the linear operator acting on $A^{1}$ defined by

$$
T_{u}(f)=Q(u f)
$$

for functions $f \in A^{1}$. Clearly $T_{u}$ is a bounded operator on $A^{1}$. In the Hilbert space context $A^{2}$, the original Toeplitz operators are defined in terms of the Bergman projection acting on $L^{2}$. But since the Bergman projection is unbounded on $L^{1}$, we

Received 4th September, 1995

This paper was partially supported by Non directed research fund, K.R.F., 1996.

Copyright Clearance Centre, Inc. Serial-fee code: 0004-9729/96 \$A2.00+0.00. 
natually consider the bounded projection $Q$ on $L^{1}$ to define the corresponding Toeplitz type operators on $A^{1}$.

A function $u \in C^{2}(B)$ is said to be pluriharmonic if its restriction to an arbitrary complex line that intersects the ball is harmonic as a function of single complex variable. As is well known [6, Theorem 4.4.9], a real-valued function on $B$ is pluriharmonic if and only if it is the real part of a holomorphic function on $B$. It follows that every pluriharmonic function on $B$ can be expressed, uniquely up to an additive constant, as the sum of a holomorphic function and an antiholomorphic function.

In the present paper, we consider a characterisation problem of two pluriharmonic symbols for which the associate Toeplitz type operators commute on $A^{1}$. In the Hilbert space case $A^{2}$, the corresponding commuting problem for the original Toeplitz operators was studied in $[1]$ with harmonic symbols on the unit disk and in $[4,7]$ with pluriharmonic symbols on the ball. The following theorem is the main result of the present paper.

THEOREM 1. Let $u$ and $v$ be bounded pluriharmonic symbols on $B$. Then $T_{u} T_{v}=T_{v} T_{u}$ on $A^{1}$ if and only if one of the following properties holds:

(a) $\quad u$ and $v$ are both holomorphic on $B$;

(b) $\quad u$ and $v$ are both antiholomorphic on $B$;

(c) there exist constants $\alpha$ and $\beta$, not both 0 , such that $\alpha u+\beta v$ is constant on $B$.

In the course of the proof of Theorem 1, we shall use an idea in [4] to give a slight variant of the characterisation of $\mathcal{M}$-harmonicity given by the weighted area version of the invariant mean value property (see Section 2 for relevent definitions) and a recent result in [7] on $\mathcal{M}$-harmonic products to characterise pluriharmonic symbols of commuting Toeplitz type operators. In Section 2 we collect some facts on $\mathcal{M}$-harmonic functions and then give a characterisation for $\mathcal{M}$-harmonic functions in terms of a weighted area version of the invariant mean value property. The characterisation will be used in Section 3 where we prove Theorem 1 and give a simple application.

\section{M-harmonic Functions}

For $z, w \in B, z \neq 0$, define

$$
\varphi_{z}(w)=\frac{z-|z|^{-2}\langle w, z\rangle z-\sqrt{1-|z|^{2}}\left(w-|z|^{-2}\langle w, z\rangle z\right)}{1-\langle w, z\rangle}
$$

and $\varphi_{0}(w)=-w$. Then $\varphi_{z} \in \mathcal{M}$, the group of all automorphisms (=biholomorphic self-maps) of $B$ and $\varphi_{z}$ is an involution: $\varphi_{z} \circ \varphi_{z}$ is the identity on $B$. Furthermore, 
each $\varphi \in \mathcal{M}$ has a unique representation $\varphi=U \circ \varphi_{z}$ for some $z \in B$ and $U \in \mathcal{U}$, the group of all unitary operators on $\mathbb{C}^{n}$. Then the real Jacobian $J_{R} \varphi$ of $\varphi$ is given by

$$
J_{R} \varphi(w)=\left(\frac{1-|z|^{2}}{|1-\langle w, z\rangle|^{2}}\right)^{n+1} \quad(w \in B)
$$

and the useful identity

$$
1-\langle\varphi(a), \varphi(b)\rangle=\frac{\left(1-|z|^{2}\right)(1-\langle a, b\rangle)}{(1-\langle a, z\rangle)(1-\langle z, b\rangle)}
$$

holds for every $a, b \in B$. See [6, Chapter 2] for details. For $u \in C^{2}(B)$ and $z \in B$, we define

$$
(\tilde{\Delta} u)(z)=\Delta\left(u \circ \varphi_{z}\right)(0)
$$

where $\Delta$ denotes the ordinary Laplacian. The operator $\tilde{\Delta}$ is called the invariant Laplacian because it commutes with automorphisms of $B$ in the sense that $\tilde{\Delta}(u \circ \varphi)=(\tilde{\Delta} u) \circ \varphi$ for $\varphi \in \mathcal{M}$. We say that a function $u \in C^{2}(B)$ is $\mathcal{M}$ - harmonic on $B$ if it is annihilated on $B$ by $\widetilde{\Delta}$. As is the case for harmonic functions on the disk, $\mathcal{M}$-harmonic functions are characterised by a certain mean value property (see [6, Theorem 4.2.4]): A function $u \in C(B)$ is $\mathcal{M}$-harmonic on $B$ if and only if

$$
(u \circ \varphi)(0)=\int_{S}(u \circ \varphi)(r \zeta) d \sigma(\zeta) \quad(0 \leqslant r<1)
$$

for every $\varphi \in \mathcal{M}$. Here $\sigma$ denotes the rotation invariant probability measure on the unit sphere $S$, the boundary of $B$. This is the so-called invariant mean value property. The following weighted area version of this invariant mean value property also gives a characterisation for $\mathcal{M}$-harmonicity of functions continuous up to $S$. In the case $\alpha=0$ the following characterisation was obtained in [6, Proposition 13.4.4], [5, Corollary 3.5] and recently with bounded function in [3] on the ball. The case $\alpha>-1$ was proved in [2, Proposition 10.2] on the disk. We now have the ball version where the case $\alpha=n+1$ will be used in the course of the proof.

Proposition 2. Let $u \in C(B)$ and $\alpha>-1$. Then $u$ is $\mathcal{M}$-harmonic on $B$ if and only if

$$
(u \circ \varphi)(0)=\lambda_{\alpha} \int_{B}(u \circ \varphi)(w)\left(1-|w|^{2}\right)^{\alpha} d V(w)
$$

for every $\varphi \in \mathcal{M}$. Here and elsewhere $1 / \lambda_{\alpha}=\int_{B}\left(1-|w|^{2}\right)^{\alpha} d V(w)$. 
ProOf: First suppose $u$ is $\mathcal{M}$-harmonic and let $\varphi \in \mathcal{M}$. By the invariant mean value property, one obtains

$$
(u \circ \varphi)(0)=\int_{S}(u \circ \varphi)(r \zeta) d \sigma(\zeta)
$$

for every $0 \leqslant r<1$. Multiplying both sides by $2 n r^{2 n-1}\left(1-r^{2}\right)^{\alpha}$ and then integrating in polar coordinates, we get

$$
(u \circ \varphi)(0) \int_{B}\left(1-|w|^{2}\right)^{\alpha} d V(w)=\int_{B}(u \circ \varphi)(w)\left(1-|w|^{2}\right)^{\alpha} d V(w)
$$

so we have (4). To prove the converse implication, we may assume $u$ is real without loss of generality, and let $U$ be the $\mathcal{M}$-harmonic function which is the invariant Poisson integral of the restriction of $u$ to $S$. Put $h=U-u$. Then $h \in C(B)$ and $h=0$ on $S$ (See [6, Chapter 3] for related facts.) Let $m$ be the maximun of $h$ on $\bar{B}$ and suppose $h\left(z_{0}\right)=m$ for some $z_{0} \in B$. Note that (4) holds for $h$. By a change of variables, (2) and (3), one obtains

$$
\begin{aligned}
h(z) & =\left(h \circ \varphi_{z}\right)(0) \\
& =\lambda_{\alpha} \int_{B}\left(h \circ \varphi_{z}\right)(w)\left(1-|w|^{2}\right)^{\alpha} d V(w) \\
& =\lambda_{\alpha} \int_{B} h(w)\left(1-\left|\varphi_{z}(w)\right|^{2}\right)^{\alpha}\left(\frac{1-|z|^{2}}{|1-\langle w, z\rangle|^{2}}\right)^{n+1} d V(w) \\
& =\lambda_{\alpha} \int_{B} h(w) \frac{\left(1-|w|^{2}\right)^{\alpha}\left(1-|z|^{2}\right)^{n+1+\alpha}}{|1-\langle w, z\rangle|^{2 n+2+2 \alpha}} d V(w)
\end{aligned}
$$

for every $z \in B$. On the other hand, by a change of variables and (2), one can easily see that

$$
I_{z}(w) d V(w)=\lambda_{\alpha}\left(1-|w|^{2}\right)^{\alpha}\left(\frac{1-|z|^{2}}{|1-\langle w, z\rangle|^{2}}\right)^{n+1+\alpha} d V(w)
$$

is a probability measure on $B$ for every $z \in B$. Since $u$ is real, we have by the above observations

$$
\begin{aligned}
m-h(z) & =h\left(z_{0}\right)-h(z) \\
& =\int_{B} h\left(I_{z_{0}}-I_{z}\right) d V \\
& \leqslant m \int_{B}\left(I_{z_{0}}-I_{z}\right) d V \\
& =0
\end{aligned}
$$


for every $z \in B$, which implies that $h=m$ on $B$. It follows that $h=0$ on $B$ because $h=0$ on $S$. Hence $u=U$, so that $u$ is $\mathcal{M}$-harmonic on $B$. The proof is complete.

The key step in our proof of Theorem 1 is adapted from that of [4]. That is, to characterise pluriharmonic symbols of commuting Toeplitz type operators, we shall use a slight variant of the characterisation of $\mathcal{M}$-harmonicity given by the weighted area version of invariant mean value property. To state it, let us introduce some notation. We associate with each $v \in C(B)$ its so-called radialisation $\mathcal{A}(v)$ defined by the formula

$$
\mathcal{A}(v)(z)=\int_{\mathcal{U}} v(U z) d U \quad(z \in B)
$$

where $d U$ denotes Haar measure on $\mathcal{U}$. Using Proposition 1.4.7 of [6], one can easily verify that

$$
\mathcal{A}(v)(z)=\int_{S} v(|z| \zeta) d \sigma(\zeta) \quad(z \in B)
$$

and hence $\mathcal{A}(v)$ is indeed a radial function on $B$. We write $\mathcal{A}(v) \in C(\bar{B})$ if $\mathcal{A}(v)$ has a continuous extension up to the boundary $S$. The following proposition was proved in Proposition 4 of $[4]$ in the case $\alpha=0$.

Proposition 3. Let $u \in C(B), \alpha>-1$ and suppose

$$
\int_{B}|u(z)|\left(1-|z|^{2}\right)^{\alpha} d V(z)<\infty
$$

Then $u$ is $\mathcal{M}$-harmonic on $B$ if and only if

$$
(u \circ \varphi)(0)=\lambda_{\alpha} \int_{B}(u \circ \varphi)(w)\left(1-|w|^{2}\right)^{\alpha} d V(w)
$$

and

$$
\mathcal{A}(u \circ \varphi) \in C(B)
$$

for every $\varphi \in \mathcal{M}$.

ProOf: We first prove the easy direction. Suppose that $u$ is $\mathcal{M}$-harmonic on $B$ and let $\varphi \in \mathcal{M}$. By the invariant mean value property again, we have

$$
(u \circ \varphi)(0)=\int_{S}(u \circ \varphi)(r \zeta) d \sigma(\zeta)
$$

for every $r \in[0,1)$. Then (5) follows by the same argument as in Proposition 2. Also (7) shows that $\mathcal{A}(u \circ \varphi)$ is constant on $B$, with value $(u \circ \varphi)(0)$, and therefore (6) holds. 
To prove the other direction (which we need for the proof of Theorem 1 with $\alpha=n+1)$, suppose that (5) and (6) hold. Let $\varphi \in \mathcal{M}$ and put $v=\mathcal{A}(u \circ \varphi)$. We first show that $v$ is $\mathcal{M}$-harmonic on $B$. Since $v \in C(B)$ by $(6)$, it is sufficient by Proposition 2 to show (4) for $v$. To do this, fix $\psi \in \mathcal{M}$. Then

$$
\lambda_{\alpha} \int_{B}(v \circ \psi)(z)\left(1-|z|^{2}\right)^{\alpha} d V=\lambda_{\alpha} \int_{B} \int_{\mathcal{U}}\left(u \circ F_{U}\right)(z)\left(1-|z|^{2}\right)^{\alpha} d U d V(z)
$$

where $F_{U}=\varphi \circ U \circ \psi \in \mathcal{M}$.

For a fixed unitary operator $U \in \mathcal{U}$, consider the inverse mapping $G_{U} \in \mathcal{M}$ of $F_{U}$ and put $a=F_{U}(0)=(\varphi \circ U \circ \psi)(0)$. Then, since $\left|\varphi^{-1}(0)\right|=|\varphi(0)|$, we have by (3)

$$
1-|a|^{2}=\frac{\left(1-|\varphi(0)|^{2}\right)\left(1-|\psi(0)|^{2}\right)}{\left|1-\left\langle\varphi^{-1}(0),(U \circ \psi)(0)\right\rangle\right|^{2}} \geqslant\left(1-|\varphi(0)|^{2}\right)\left(1-|\psi(0)|^{2}\right) .
$$

On the other hand, we have by (3) again

$$
1-\left|G_{U}(w)\right|^{2}=\frac{\left(1-|a|^{2}\right)\left(1-|w|^{2}\right)}{|1-\langle w, a\rangle|^{2}}
$$

and by (2)

$$
J_{\mathrm{R}} G_{U}(w)=\left(\frac{1-|a|^{2}}{|1-\langle w, a\rangle|^{2}}\right)^{n+1}
$$

for every $w \in B$. It follows that

$$
\begin{aligned}
\left(1-\left|G_{U}(w)\right|^{2}\right)^{\alpha} J_{\mathrm{R}} G_{U}(w) & =\frac{\left(1-|a|^{2}\right)^{n+1+\alpha}\left(1-|w|^{2}\right)^{\alpha}}{|1-\langle w, a\rangle|^{2(n+1+\alpha)}} \\
& \leqslant\left(1-|w|^{2}\right)^{\alpha}\left(\frac{4}{1-|a|^{2}}\right)^{n+1+\alpha} \quad(w \in B) .
\end{aligned}
$$

Now a change of variables and the above, together with (9) show

$$
\begin{aligned}
\int_{U} \int_{B}\left|u \circ F_{U}(z)\right| & \left(1-|z|^{2}\right)^{\alpha} d V(z) d U \\
& =\int_{U} \int_{B}|u|\left(1-\left|G_{U}\right|^{2}\right)^{\alpha} J_{\mathrm{R}} G_{U} d V d U \\
& <\infty
\end{aligned}
$$


since $\int_{B}|u(z)|\left(1-|z|^{2}\right)^{\alpha} d V(z)<\infty$ by assumption. Now one can interchange the order of integrations on the right side of $(8)$ to obtain

$$
\begin{aligned}
\lambda_{\alpha} \int_{B}(v \circ \psi)(z)\left(1-|z|^{2}\right)^{\alpha} d V(z) & =\lambda_{\alpha} \int_{\mathcal{U}} \int_{B}\left(u \circ F_{U}\right)(z)\left(1-|z|^{2}\right)^{\alpha} d V(z) d U \\
& =\int_{\mathcal{U}}\left(u \circ F_{U}\right)(0) d U \\
& =\int_{\mathcal{U}}(u \circ \varphi \circ U)(\psi(0)) d U \\
& =\mathcal{A}(u \circ \varphi)(\psi(0)) \\
& =(v \circ \psi)(0)
\end{aligned}
$$

where the second equality holds by (5). Hence $v$ is $\mathcal{M}$-harmonic on $B$. Since $v$ is radial, the invariant mean value property shows that $v$ is constant. Consequently,

$$
(u \circ \varphi)(0)=v(0)=v(z)=\int_{S}(u \circ \varphi)(|z| \zeta) d \sigma(\zeta) \quad(z \in B) .
$$

Since $\varphi \in \mathcal{M}$ is arbitrary, the above shows that $u$ has the invariant mean value property and hence that $u$ is $\mathcal{M}$-harmonic on $B$ as desired. The proof is complete.

Before turning to the our proof, we need a recent result of Zheng [7] on $\mathcal{M}$-harmonic products to characterise the symbols. (The original statement in [7, Theorem 2] is in a slightly different form.)

LEMMA 4. Let $u=f+\bar{g}$ and $v=h+\bar{k}$ be two bounded pluriharmonic symbols on $B$. If $f \bar{k}-h \bar{g}$ is $\mathcal{M}$-harmonic on $B$, then $u$ and $v$ are all holomorphic or antiholomorphic or there exist constants $\alpha$ and $\beta$, not both 0 , such that $\alpha u+\beta v$ is constant on $B$.

\section{ProOF}

First, we recall some well known facts on the Hardy space $H^{2}$ consisting of holomorphic functions $f$ on $B$ for which

$$
\sup _{0<r<1} \int_{S}|f(r \zeta)|^{2} d \sigma(\zeta)<\infty
$$

Note that $H^{2} \subset A^{2}$ by an integration in polar coordinates. In addition, it is shown in [4] that $\mathcal{A}(f \bar{g}) \in C(B)$ for every $f, g \in H^{2}$.

Next, before turning to the proof of Theorem 1, we prove a couple of lemmas. For $\varphi \in \mathcal{M}$, let $U_{\varphi}$ denote the linear operator on $L^{1}$ defined by

$$
U_{\varphi} f=(f \circ \varphi)(J \varphi)^{2}
$$


where $J \varphi$ is the complex Jacobian of $\varphi$. Since $|J \varphi|^{2}$ is the real Jacobian of $\varphi$, one obtains by a change of variables

$$
\int_{B}\left|U_{\varphi} f\right| d V=\int_{B}|f \circ \varphi||J \varphi|^{2} d V=\int_{B}|f| d V
$$

for every $f \in L^{1}$. Hence $U_{\varphi}$ is an isometry of $L^{1}$ into $L^{1}$ and clearly $U_{\varphi}$ takes $A^{1}$ onto $A^{1}$. Moreover it is easy to see that $U_{\varphi} U_{\varphi^{-1}}=U_{\varphi^{-1}} U_{\varphi}$ is the identity operator on $L^{1}$. The following lemma is essentially contained in [8] (in a slightly different case). But we here give a proof for the sake of completeness.

Lemma 5. Let $\varphi \in \mathcal{M}$. Then $Q U_{\varphi}=U_{\varphi} Q$ on $L^{1}$.

Proof: Let $\varphi \in \mathcal{M}$ with the representation $\varphi^{-1}=U \circ \varphi_{a}$ for some $a \in B$ and $U \in \mathcal{U}$. Note by $[8$, Section 2$]$ that

$$
\left(J \varphi_{a}\right)(z)=(-1)^{n}\left(\frac{\sqrt{1-|a|^{2}}}{1-\langle z, a\rangle}\right)^{n+1} \quad(z \in B) .
$$

It follows from a straightforward calculation that

$$
(J \varphi)^{2}\left(\varphi^{-1}(z)\right)=\frac{(1-\langle z, a\rangle)^{2 n+2}}{\left(1-|a|^{2}\right)^{n+1}} \quad(z \in B) .
$$

Let $f \in L^{1}$ and pick a point $z \in B$. By a change of variables and a simple manipulation using (2) and (3), one can see from (11) that

$$
\begin{aligned}
Q\left(U_{\varphi} f\right)(z) & =\lambda_{n} \int_{B} \frac{\left(1-|w|^{2}\right)^{n+1}}{(1-\langle z, w\rangle)^{2 n+2}}(f \circ \varphi)(w)(J \varphi)^{2}(w) d V(w) \\
& =\lambda_{n} \int_{B} \frac{\left(1-\left|\varphi^{-1}(w)\right|^{2}\right)^{n+1}}{\left(1-\left\langle z, \varphi^{-1}(w)\right\rangle\right)^{2 n+2}} f(w)(J \varphi)^{2}\left(\varphi^{-1}(w)\right) J_{R} \varphi^{-1}(w) d V(w) \\
& =\lambda_{n} \frac{\left(1-|a|^{2}\right)^{n+1}}{(1-\langle z, U a\rangle)^{2 n+2}} \int_{B} \frac{\left(1-|w|^{2}\right)^{n+1}}{\left(1-\left\langle\varphi_{a} U^{-1}(z), w\right\rangle\right)^{2 n+2}} f(w) d V(w) .
\end{aligned}
$$

On the other hand, (10) shows that the last expression of the above is just the same as $(J \varphi)^{2}(z) Q f(\varphi(z))$, which is exactly $U_{\varphi} Q f(z)$. Hence $U_{\varphi} Q=Q U_{\varphi}$ on $L^{1}$, as desired. The proof is complete.

Lemma 6. Let $\varphi \in \mathcal{M}$ and $u \in L^{\infty}$. Then

$$
U_{\varphi} T_{u} U_{\varphi^{-1}}=T_{u \circ \varphi}
$$


Proof: Let $f \in A^{1}$. By Lemma 5, one obtains

$$
\begin{aligned}
T_{u \circ \varphi} U_{\varphi} f & =T_{u \circ \varphi}\left[(f \circ \varphi)(J \varphi)^{2}\right]=Q\left[(u \circ \varphi)(f \circ \varphi)(J \varphi)^{2}\right] \\
& =Q U_{\varphi}(u f)=U_{\varphi} Q(u f)=U_{\varphi} T_{u} f
\end{aligned}
$$

Thus $T_{u \circ \varphi} U_{\varphi}=U_{\varphi} T_{u}$ on $L^{1}$. Now use the fact $U_{\varphi} U_{\varphi^{-1}}$ is the identity operator to get the desired result. This completes the proof.

We are now ready to prove Theorem 1 .

Proof of Theorem 1: We begin with the easy direction. First suppose that (a) holds, so that $u$ and $v$ are holomorphic on $B$, which means that $T_{u}$ and $T_{v}$ are, respectively, the operators on $A^{1}$ of multiplication by $u$ and by $v$ by (1). Thus $T_{u} T_{v}=T_{v} T_{u}$ on $L^{1}$. Now assume $(b)$, so that $\bar{u}$ and $\bar{v}$ are holomorphic on $B$. By the explict formula for the operator $Q$ and an application of Fubini's theorem, one can see that $T_{u} T_{v} f=Q(u v f)$ for every bounded function $f$ in $A^{1}$. Note that the set of all bounded functions in $A^{1}$ forms a dense subset of $A^{1}$. It follows from continuity that $T_{u}$ and $T_{v}$ commute, as desired. Finally suppose $(c)$ holds and assume $\alpha \neq 0$ (the other case is similar). Then $u=c_{1} v+c_{2}$ for some constants $c_{1}$ and $c_{2}$, which implies $T_{u}=c_{1} T_{v}+c_{2}$, so that $T_{u} T_{v}=c_{1} T_{v} T_{v}+c_{2} T_{v}=T_{v} T_{u}$ on $A^{1}$.

Now we prove the converse implication. Write $u=f+\bar{g}$ and $v=h+\bar{k}$ for some holomorphic $f, g, h$, and $k$. It is shown in the proof of Theorem 1 in [4] that functions $f, g, h$, and $k$ are all in $H^{2}$. Since $H^{2} \subset A^{2} \subset A^{1}$, in particular, functions $f, g, h$, and $k$ are all in $A^{1}$. Let 1 denote the constant function 1 on $B$. Then we have by (1)

$$
\begin{aligned}
T_{u} T_{v} 1 & =T_{u}(Q v)=T_{u}(h+\bar{k}(0)) \\
& =Q(f h+\bar{k}(0) f+h \bar{g}+\bar{g} \bar{k}(0)) \\
& =f h+\bar{k}(0) f+Q(h \bar{g})+\bar{g}(0) \bar{k}(0)
\end{aligned}
$$

Note that $\int_{B} F d V=F(0)$ for holomorphic functions $F \in L^{1}$. It follows that

$$
\begin{aligned}
\int_{B}\left(T_{u} T_{v} 1\right) d V= & \left(T_{u} T_{v} 1\right)(0) \\
= & f(0) h(0)+f(0) \bar{k}(0)+\bar{g}(0) \bar{k}(0)+Q(h \bar{g})(0) \\
= & f(0) h(0)+f(0) \bar{k}(0)+\bar{g}(0) \bar{k}(0) \\
& \quad+\lambda_{n} \int_{B} h(w) \bar{g}(w)\left(1-|w|^{2}\right)^{n+1} d V(w)
\end{aligned}
$$

Similarly,

$\int_{B}\left(T_{v} T_{u} 1\right) d V=f(0) h(0)+h(0) \bar{g}(0)+\bar{g}(0) \bar{k}(0)+\lambda_{n} \int_{B} f(w) \bar{k}(w)\left(1-|w|^{2}\right)^{n+1} d V(w)$ 
Since $T_{u} T_{v}=T_{v} T_{u}$ by assumption, letting $\delta=f \bar{k}-h \bar{g}$, we have by (12) and (13) that

$$
\lambda_{n} \int_{B} \delta(w)\left(1-|w|^{2}\right)^{n+1} d V(w)=\delta(0) .
$$

We also have (by a remark mentioned at the beginning of this section) that

$$
\mathcal{A}(\delta) \in C(B)
$$

Let $\varphi \in \mathcal{M}$. Multiplying both sides of the equation $T_{u} T_{v}=T_{v} T_{u}$ by $U_{\varphi}$ on the left and by $U_{\varphi^{-1}}$ on the right, we obtain since $U_{\varphi^{-1}} U_{\varphi}$ is the identity operator that

$$
U_{\varphi} T_{u} U_{\varphi^{-1}} U_{\varphi} T_{v} U_{\varphi^{-1}}=U_{\varphi} T_{v} U_{\varphi^{-1}} U_{\varphi} T_{u} U_{\varphi^{-1}}
$$

and therefore by Lemma 6

$$
T_{u \circ \varphi} T_{v \circ \varphi}=T_{v \circ \varphi} T_{u \circ \varphi}
$$

Equations (14) and (15) were derived under the assumption that $T_{u} T_{v}=T_{v} T_{u}$. Thus (16) says that (14) and (15) remain valid with $\delta \circ \varphi$ in place of $\delta$. That is,

$$
\lambda_{n} \int_{B}(\delta \circ \varphi)(w)\left(1-|w|^{2}\right)^{n+1} d V(w)=(\delta \circ \varphi)(0)
$$

and $\mathcal{A}(\delta \circ \varphi) \in C(\bar{B})$ for any $\varphi \in \mathcal{M}$. It follows from Proposition 3 with $\alpha=n+1$ that $\delta=f \bar{k}-h \bar{g}$ is $\mathcal{M}$-harmonic on $B$. Now Lemma 4 gives the desired characterisation. This completes the proof.

We conclude this paper with a simple application. We note that pluriharmonic functions are closed under complex conjugation.

COROLlaRY 7. Let $u$ be a bounded pluriharmonic symbol on $B$. Then $T_{u} T_{\bar{u}}=$ $T_{\bar{u}} T_{u}$ on $A^{1}$ if and only if the image of $B$ under $u$ lies on some line in $\mathbb{C}$.

Proof: If $u(B)$ lies on some line in $\mathbb{C}$, a rotation and a translation show that there exist constants $c(|c|=1)$ and $d$ such that $c u+d$ is real valued on $B$. Since $T_{u}=\left(T_{c u+d}-d\right) / c$ and $T_{\bar{u}}=\left(T_{c u+d}-\bar{d}\right) / \bar{c}$, one can show that $T_{u}$ and $T_{\bar{u}}$ commute. Conversely assume $T_{u} T_{\bar{u}}=T_{\bar{u}} T_{u}$ on $A^{1}$ and then Theorem 1 implies that $u$ and $\bar{u}$ are holomorphic on $B$ or a nontrivial linear combination of $u$ and $\bar{u}$ is constant on $B$. The first case implies $u$ is constant on $B$, so we are done. Also, a simple manipulation shows that the latter case implies $u(B)$ lies on some line in $\mathbb{C}$. This completes the proof. 


\section{References}

[1] S. Axler and Ž. Cučković, 'Commuting Toeplitz operators with harmonic symbols', Integral Equations Operation Theory 14 (1991), 1-11.

[2] J. Arazy, S.D. Fisher and J. Peetre, 'Hankel operators on weighted Bergman spaces', Amer. J. Math. 110 (1988), 989-1054.

[3] P. Ahern, M. Flores and W. Rudin, 'An invariant volume-mean-value property', J. Funct. Anal. 111 (1993), 380-397.

[4] B.R. Choe and Y.J. Lee, 'Pluriharmonic symbols of commuting Toeplitz operators', Illinois J. Math. 37 (1993), 424-436.

[5] K.T. Hahn and E.H. Youssfi, ' $\mathcal{M}$-harmonic Besov p-spaces and Hankel operators in the Bergman spaces on the unit ball in $\mathbb{C}^{n}$, Manuscripta Math. 71 (1991), 67-81.

[6] W. Rudin, Function theory in the unit ball of $\mathbb{C}^{n}$ (Springer-Verlag, Berlin, Heidelberg, New York, 1980).

[7] D. Zheng, 'Commuting Toeplitz opetators with pluriharmonic symbols', (preprint).

[8] K. Zhu, 'Hankel-Toeplitz type operators on $L_{a}^{1}(\Omega)$ ', Integral Equations Operation Theory 13 (1990), 285-302.

Department of Mathematics

College of Natural Sciences

Mokpo National University

Choinnam 534-729

Korea 